\title{
Las Tecnologías de la Información y la Comunicación y su Influencia en la Transformación de la Educación Superior en Colombia para Impulso de la Economía Global
}

\author{
Emiro De-la-Hoz-Franco ${ }^{1}$, Olga Martínez-Palmera ${ }^{2}$, Harold Combita-Niño y Hugo Hernández-Palma ${ }^{3}$ \\ (1) Dpto. de Ciencias de la Computación y Electrónica, Universidad de la Costa, Barranquilla-Colombia \\ (2) Dpto. de Humanidades, Universidad de la Costa, Barranquilla-Colombia \\ (3) Programa Administración de Empresas, Universidad del Atlántico, Barranquilla-Colombia \\ E-mail: edelahoz@cuc.edu.co
}

Recibido Abr. 26, 2018; Aceptado Jul. 4, 2018; Versión final Sep. 11, 2018, Publicado Feb. 2019

\begin{abstract}
Resumen
En este artículo se reflexiona desde el punto de vista conceptual, sobre la influencia que las Tecnologías de la Información y la Comunicación (TIC) y el impacto que éstas han generado en los procesos de enseñanza/aprendizaje. La educación superior en Colombia ha venido transformándose en las recientes décadas, con miras a responder de manera decidida a los requerimientos de una sociedad renovada, que busca contar con profesionales más hábiles, que gestionen eficientemente procesos administrativos y académicos en Instituciones de Educación Superior en estos tiempos de globalización económica. La metodología utilizada en este estudio se enmarca en la revisión documental de investigaciones y publicaciones efectuadas en los últimos diez años. Los resultados apuntan a que las TIC han aportado considerablemente a diferentes aspectos neurálgicos, en el contexto educativo y que las aplicaciones en los procesos pedagógicos son cada vez mayores.
\end{abstract}

Palabras clave: tecnologías de la información y la comunicación; educación superior; economía; globalización

\section{Information and Communication Technologies and their Influence on the Transformation of Higher Education in Colombia to Boost the Global Economy}

\begin{abstract}
This document presents a conceptual analysis on the influence of Information and Communication Technologies (ICT) and the impact they have generated in the teaching / learning processes. Higher education in Colombia has been transformed in recent decades, with the aim of decisively responding to the requirements of a renewed society, which seeks to have more skilled professionals, who efficiently manage administrative and academic processes in Higher Education Institutions in these times of economic globalization. The methodology used is part of the documentary review of research and publications carried out during the last ten years. The results indicate that ICT have significantly contributed to different neuralgic aspects, in the educational context and that applications in pedagogical processes are increasing.
\end{abstract}




\section{INTRODUCCIÓN}

La sociedad actual, caracterizada por un contexto de globalización y por el rápido crecimiento de las Tecnologías de la Información y la Comunicación (TIC), ha impulsado la creación de mecanismos para la incorporación de diferentes avances tecnológicos en variados sectores económicos y sociales, fortaleciendo tales escenarios en cuanto a su eficiencia y dinamismo (Baller et al., 2016). El sector educativo no escapa a esta realidad, incorporando las TIC como fenómeno inminente y de alta incidencia en el contexto educativo, en atención a la necesidad de promover la innovación y creatividad como mecanismo de competencia (Sanz et al., 2012), en el que se deben enmarcar las Instituciones de Educación Superior (IES), para la operacionalización de sus funciones sustantivas. Consecuente con ello, el sistema de enseñanza-aprendizaje tiende a adaptarse a estos cambios, a través de la creación de políticas educativas que integren estas herramientas en los entornos de aprendizaje (Herrera, 2015). En la literatura se encuentran diversos enfoques para determinar a nivel conceptual, ¿qué son las TIC?; desde una perspectiva técnica, las TIC se fundamentan en: los referentes relativos a las tecnologías de la comunicación (medios de radiodifusión, televisión y telefonía, entre otros) y las tecnologías de la información, que engloban los mecanismos en los cuales se almacenan y gestionan datos e información (Ausín et al., 2016).

En un sentido global, las TIC corresponden a los mecanismos y herramientas mediante los cuales se puede procesar, almacenar, distribuir y difundir información de diversas fuentes. Constituyéndose en un elemento distintivo de la innovación que caracteriza a la sociedad moderna y que está influyendo en el aprendizaje de los individuos y en las formas tradicionales para la difusión del conocimiento (Zempoalteca et al., 2016). Específicamente, en el contexto educativo las TIC corresponden a un conjunto de herramientas tecnológicas de apoyo al docente para el fortalecimiento del proceso de enseñanza-aprendizaje en todos los niveles de la educación (Arancibia et al., 2010).

Según la Organización de las Naciones Unidas para la Educación, la Ciencia y la Cultura (UNESCO), la incorporación de las TIC en la educación superior se emplea en la elaboración de material didáctico, compartir contenidos, mejorar la comunicación entre los actores del proceso educativo, promover y desarrollar la investigación y facilitar los procesos administrativos institucionales, entre otros (UNESCO, 2013). En la mayoría de las IES se ha adoptado a las TIC como un medio para la transformación y mejoramiento de los procesos de enseñanza-aprendizaje en el sistema educativo, previa concientización de sus limitaciones y factores aprovechables (Koh et al., 2015). Sin embargo, el uso e implementación de las TIC no es sinónimo de éxito garantizado en cuanto al desarrollo de mecanismos eficientes de creación y difusión del conocimiento. Por tal razón, es importante promover espacios con las condiciones adecuadas para el máximo aprovechamiento de estas herramientas (De Witte y Rogge, 2014).

\section{METODOLOGÍA}

En este estudio se efectuó una revisión detallada de las publicaciones científicas en los últimos 10 años en relación al uso de las TIC en educación superior en Colombia. Igualmente se hizo una revisión exhaustiva de la literatura internacional para poner el tema y la discusión en un contexto internacional y comparar la situación actual con la de Colombia. La compilación de la información se realizó de manera organizada y contemplando aspectos como objetividad, impacto y análisis de los conceptos articulados o vinculados a las TIC en la educación superior. Para la búsqueda de los artículos y publicaciones científicas en idioma castellano se utilizó la base de datos especializada IEEExplore, la biblioteca científica y electrónica Scielo, el sistema abierto de información de revistas Dialnet y la base de datos de revistas Científicas Redalyc, por ser los repositorios que más se especializan en publicaciones científicas relacionadas con esta tipología de investigación.

Para la literatura internacional en idioma ingles se exploró las publicaciones de revistas indizadas en la Web of Science (WoS) y en Scopus. El proceso se realizó en tres fases: ubicación de palabras clave en los buscadores sobre el origen, evolución e influencia de las TIC en la educación superior, selección de publicaciones recientes y construcción reflexiva a partir de los aspectos propuestos por los diversos investigadores en cada uno de los trabajos consultados.

\section{RESULTADOS Y DISCUSION}

Al efectuar la revisión de la literatura se ha podido evidenciar, según lo manifestado por (Bejarano et al., 2013) que el origen de las TIC es a mediados del año 1940, fundamentándose en el ámbito disciplinar de la electrónica y empleándose inicialmente en los procesos industriales y científicos. El periodo siguiente a la II guerra mundial impulso el desarrollo de un variado número de innovaciones, aplicables tanto al ámbito militar como al civil. Las más destacables han sido el transistor como elemento fundamental de la microelectrónica y en consecuencia el ordenador programable. Los avances en la electrónica dieron paso a la creación del microprocesador, a mediados de los años 70, el cual se transformó en una pieza clave para el desarrollo y 
crecimiento de la microelectrónica. De esta forma, los orígenes de las TIC están directamente vinculados al desarrollo de los ordenadores, protagonizados en ese entonces por las incipientes compañías IBM, Apple y Microsoft (Bejarano et al., 2013).

Estos avances permitieron abrir nuevos campos de innovación en los cuales, las telecomunicaciones, fueron un sector que se desarrolló de forma acelerada (Roldán et al., 2016). Evidencia de esto es que para el año de 1969 se creó el primer conmutador electrónico industrial y al siguiente año se desarrolló el digital, dándose un gran paso en este sentido (Sarduy y Felipe, 2014). Estas acciones propiciaron nuevos desarrollos, tales como la fibra óptica. Fabricada de manera industrial por Corning Glass en los años 70 y en los años siguientes el perfeccionamiento de la red de comunicación del Departamento de Defensa estadounidense denominado Advanced Research Project Agency (ARPA) que posteriormente se convertiría en lo que hoy se conoce como internet (Adell et al., 2015).

Lo anteriormente descrito cimentó las bases de desarrollos tecnológicos, tales como: la telefonía celular, los computadores personales y los dispositivos de video y audio personales, la automatización y el internet para las cosas cotidianas, entre otros (Pinto et al., 2012); los cuales fomentaron un conjunto tecnologías que hoy se conocen como TIC (Sarnou, 2015). Esto conllevó a nuevas formas de comunicación y difusión, impactando la manera como el individuo accede y obtiene la información actualmente, que en muchos casos, proviene de múltiples fuentes y formatos, generando a su vez una nueva problemática, la sobreinformación (Recalde, 2015). Así las cosas, la capacidad de responder y procesar esos grandes volúmenes de información, que se generan actualmente en las redes, es uno de los retos que el individuo debe afrontar. Atendiendo a la calidad y veracidad de la información, la mayor dificultad se encuentra en su utilización y disposición en un contexto lógico, que permita su transformación en verdadero conocimiento (Hernández et al., 2017).

\section{Las TIC en el mundo}

La sociedad a nivel global se ha visto impactada de manera notoria por el efecto generado por las TIC en todas sus dimensiones; las transformaciones sociales, económicas, educacionales y culturales han posibilitado nuevos escenarios donde el individuo ha potenciado todos los aspectos que se ha propuesto en búsqueda de un panorama más confortable y estable según sus expectativas de vida (Jin y Cho, 2015). En la misma línea, los aspectos productivos y de servicios han variado de manera vertiginosa llegando a niveles de excelencia nunca antes vistos, donde los usuarios y clientes reciben enormes beneficios derivados de los adelantos logrados tanto en tecnología como en información (Pérez et al., 2018).

Según estudios de varias metodologías realizadas por organizaciones diversas, las influencias más destacables de las TIC a nivel global tienen que ver con la universalización de las cosas, pues en la medida que las tecnologías y la difusión de la información han crecido gracias a los avances conocidos, se ha logrado conectar al mundo entero en todos los terrenos, propiciando puntos de encuentro cada vez más fluidos, dinámicos y competitivos, lo que sin duda ha fomentado el bienestar y crecimiento socio-económico para muchas comunidades (Asongu y Le-Roux, 2017).

En temas de sectores particulares son muchos los ejemplos que se pueden citar, pues desde los sistemas masivos de transporte, salud, producción de bienes y hasta los programas de educación de todo nivel, se han visto mejoras e innovaciones que han permitido acortar distancias, recortar tiempos de atención, realizar procedimientos no contemplados y promover la cercanía entre ciudades y grupos de interés, lo que sin duda ha posibilitado la apertura de una sociedad virtual donde no es indispensable la presencia física para sacar adelante los desarrollos o procesos que se requieren en ciertos aspectos (Baldassar et al., 2016).

\section{Las TIC en el contexto educativo}

En el contexto educativo, se han venido incorporando las TIC en los últimos años, con especial ímpetu específicamente en los procesos de enseñanza/aprendizaje (De la Hoz-Correa et al., 2014). Lo anterior ha desencadenado una serie de cambios en la sociedad moderna, relativos a la creación de entornos de aprendizaje menos rígidos, en los cuales el factor temporal o de ubicación espacial es prácticamente descartado (Cabero, 2010). Como se muestra en la figura 1, las TIC han llenado espacios que anteriormente no se contemplaba en la educación tradicional.

Bajo este escenario es relevante mencionar la importancia que tienen los nativos digitales, término acuñado por Prensky (2001), el cual sostiene que la estructura de pensamiento de éstos es significativamente distinta a la de aquellos que no crecieron en el entorno digital de hoy en día. Por lo tanto, esta generación de nativos digitales probablemente piense de manera distinta al resto de las generaciones anteriores. En tal sentido, para los nativos digitales la experiencia en el uso de las TIC crea nuevas demandas en los procesos de aprendizaje, que el sistema educativo se esfuerza por atender. El estudiante contemporáneo usa la tecnología 
como herramienta básica en el contexto personal y espera que también lo sea en el educativo (Bello, 2018). Sin embargo, no se debe esperar que la totalidad de nativos digitales sean capaces de conocer a profundidad el uso de las TIC de manera automática, dado que sus habilidades y conocimientos sobre la tecnología presenta variabilidad acorde a las características particulares de cada individuo (Boyd, 2015).

Tomando como base el hecho de que los nativos digitales poseen mayores habilidades en el uso de recursos tecnológicos, y que su forma de pensar está adaptada en cierta medida a éstos, el docente se convierte en un guía cuyo objetivo primordial es facilitar la usabilidad de los medios tecnológicos, con fines educativos provechosos. Este aprovechamiento, se puede entender, en la educación superior, como la transferencia eficiente del conocimiento que propicie en el estudiante la comprensión y generación de nuevo conocimiento (Avgerou et al., 2016).

\section{Aplicaciones de las TIC en el ámbito educativo}

Tomando en consideración los postulados hasta ahora citados, se ha podido evidenciar que las estrategias de enseñanza/aprendizaje han evolucionado a espacios de mayor flexibilidad, innovación y creatividad. Delegando en el docente la labor de diseñar nuevas formas de propiciar la apropiación del conocimiento por parte de los estudiantes y de gestionar múltiples medios de comunicación con los educandos (Albion et al., 2015). La figura 1 que se muestra seguidamente, esquematiza lo anteriormente descrito.

Como se observa en la figura 1, la integración en un mismo plano de aspectos tales como: flexibilidad, innovación y creatividad, promueven un escenario de aplicación de las estrategias pedagógicas mucho más amplio al que se manejaba hasta hace unas décadas; dinamizando los procesos asociados y brindando espacios, antes no contemplados, que facilitarán la interacción entre los diversos actores. Es importante resaltar que para el fortalecimiento de las TIC en contextos educativos, como herramientas que promueven la innovación de los procesos de enseñanza, las estrategias no sólo deben aplicarse desde el enfoque pedagógico, adicionalmente deben generarse escenarios de actuación en los que se incentive la apropiación de tecnologías en el modelo educativo, en la producción de materiales digitales, la formación de docentes para el uso significativo de las TIC y la investigación relacionada con las TIC y la Innovación, tal como se planteó en (Marín et al., 2017).

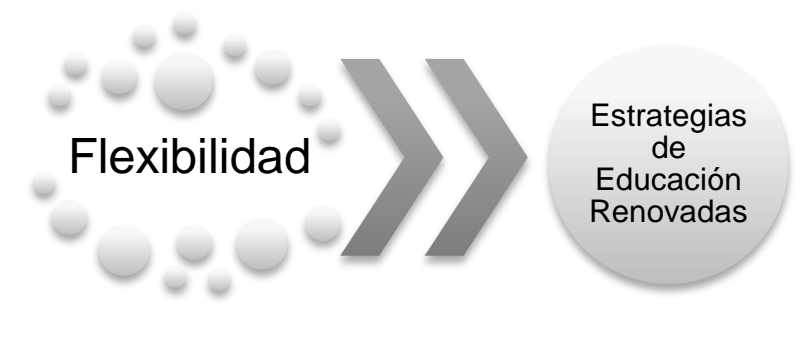

Innovación Creatividad

Fig. 1: Estrategias enseñanza/aprendizaje con mediación de TIC (tomado de Albion et al., 2015)

Las TIC ofrecen las herramientas para la creación de tales escenarios y posibilitan el diseño e implementación de nuevas estrategias pedagógicas. Ejemplo de ello, es el uso de Entornos Virtuales de Enseñanza Aprendizaje (EVEA), operacionalizados a través de plataformas virtuales, que permiten la interacción entre docentes y estudiantes alrededor de un contenido específico, y mediante la utilización de métodos y técnicas bien definidas, con la finalidad de fomentar las competencias y generar conocimiento de forma significativa (López y Hernández, 2016). En este orden de ideas, la diversidad de dispositivos que existen en la actualidad, tales como teléfonos inteligentes y tablets, fácilmente pueden ser integrados a este ecosistema digital, con el objeto de recrear espacios innovadores que estimulen el aprendizaje.

En ese sentido, el m-Learning (aprendizaje electrónico móvil), es un espacio en el cual, el estudiante aprende a través de su interacción con dispositivos móviles, dinamizando su proceso de aprendizaje en un contexto tanto real como virtual (López, 2016). Lo citado, abre paso a la posibilidad de utilizar estos dispositivos móviles para integrarlos a los llamados Entornos Personales de Aprendizaje (EPA) los cuales se pueden categorizar en EPA Pedagógico y EPA Tecnológico, como se describe en la figura 2. En coherencia con la gráfica, los EPA pedagógicos promueven el autoaprendizaje a través de la utilización de recursos web. De esta forma, el estudiante es protagonista y dueño de su proceso de aprendizaje, estableciendo sus pautas y objetivos. Por otra parte, los EPA tecnológicos, conciben el entorno de aprendizaje como una plataforma compuesta por contenidos y diversas herramientas de gestión y comunicación (Liyanagunawardena et al., 2014). 
En consecuencia, indistintamente de las corrientes antes mencionadas, los EPA promueven nuevos espacios para el aprendizaje, en los cuales el protagonista del proceso de enseñanza-aprendizaje es el estudiante. Lo cual contrasta con la metodología de enseñanza tradicional, en la que el docente es quien guía y conduce totalmente el proceso. Este conjunto de posibilidades, abre paso a nuevas estrategias para difundir el conocimiento, brindando oportunidades y espacios flexibles con diversas ventajas para el estudiante (GarcíaGómez et al., 2016). Consecuentemente, la implementación de estas innovadoras estrategias, basadas en las TIC, propicia un espacio comparativo para la reflexión a partir del análisis de las ventajas y limitantes, ver figura 2.
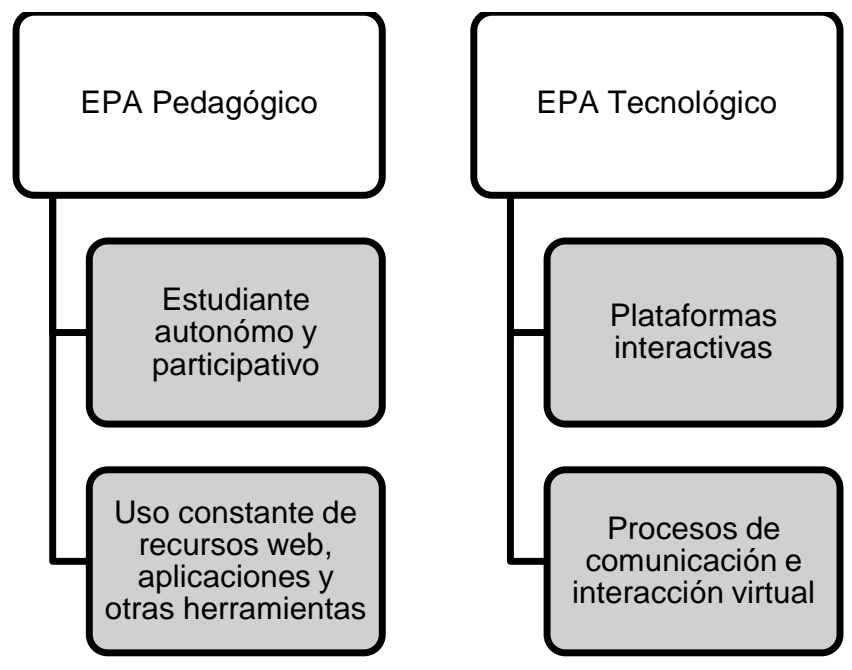

Fig. 2: Categorías de los Entornos Virtuales de Aprendizaje (Tomado de López, 2016)

La principal ventaja de la incorporación de las TIC en el ámbito educativo basado en EPA, consiste en la generación de un mayor dinamismo e interactividad entre docentes y estudiantes. En tal sentido, nuevos canales y formas de comunicación surgen entre los diferentes actores del proceso educativo, enriqueciendo y potenciando la interacción entre estos (Molina et al., 2015). Sin embargo, podría presentarse una limitación, en cuanto a la adquisición de los recursos tecnológicos móviles, que debe poseer el estudiante para acceder a los EVEA y a la curva de aprendizaje en su uso.

Pese a que se ha popularizado el uso de dispositivos móviles en los años recientes y a que las apps cada vez son más intuitivas en cuanto a sus alternativas de uso, son varios los aspectos susceptibles de análisis y revisión. Por otra parte, es complejo que las instituciones subsidien la adquisición de estos recursos (que tienen una connotación personal), dada la elevada inversión que ello supone. Es importante resaltar que no basta con la incorporación de software y equipos a este ecosistema digital, también se requiere una estrategia adecuada para su implementación en el proceso educativo (Cabero, 2010). Así las cosas, en la figura 3 se ilustran los aspectos que mayor ventaja y limitantes podrían tomarse en cuenta a partir de los lineamientos expuestos.

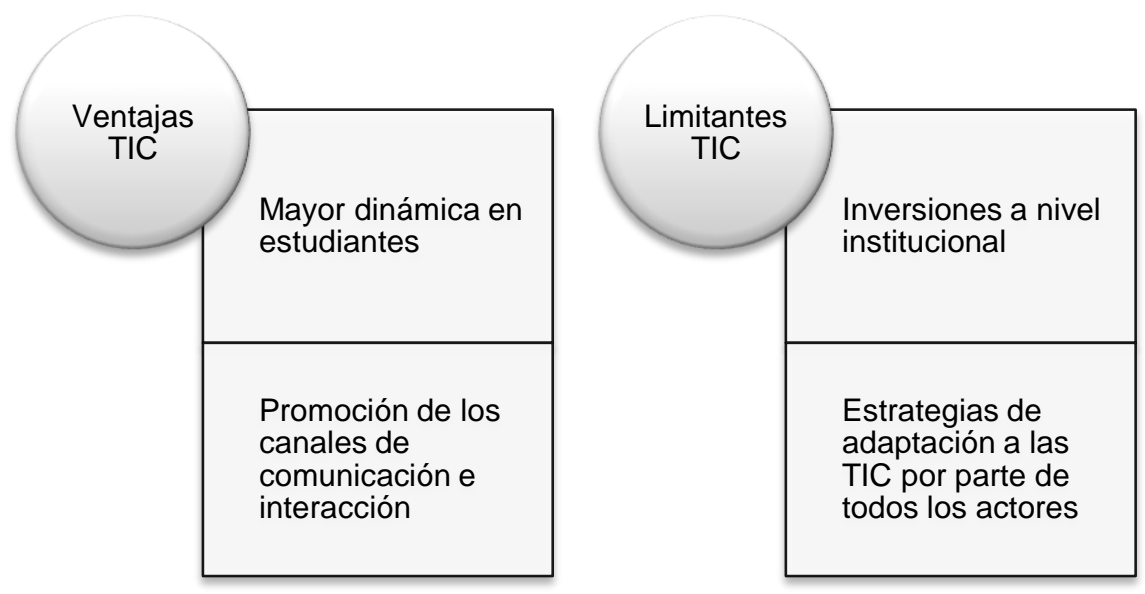

Fig. 3: Ventajas y desventajas de la adopción de las TIC basadas en EPA 
En este orden de ideas, las TIC no deben confundirse únicamente con la adquisición o uso de equipos tecnológicos que, si bien es una parte importante del proceso, implica un compromiso mayor de los actores involucrados en el acto educativo, que consiste primordialmente en generar un cambio cultural y estructural en las prácticas docentes (Mac Callum y Jeffrey, 2014). En este sentido, la evidencia de tal compromiso debe ser definida desde el diseño de las estrategias requeridas para lograr una implementación adecuada de las TIC en los procesos de enseñanza basados en EPA, integrando en las implementaciones tres elementos o componentes claves, como se aprecia en la figura 4.

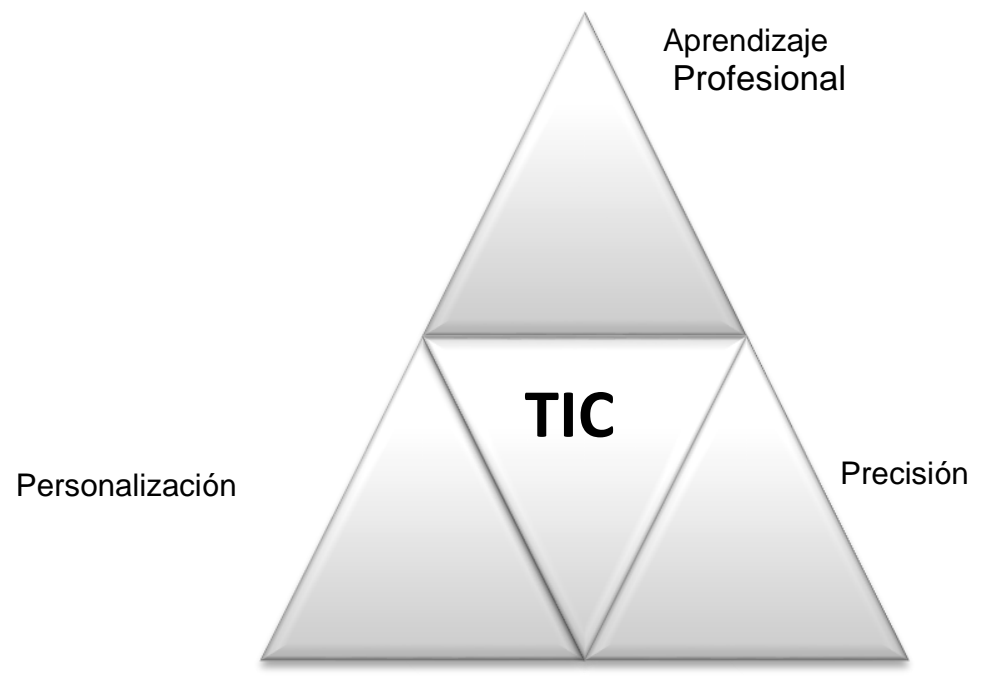

Fig. 4: Componentes requeridos para la implementación de TIC basadas en EPA

En los estudios relacionados con las TIC se describen estos tres componentes: precisión, aprendizaje profesional y personalización. En primer lugar, la precisión hace referencia al acceso y uso adecuado de los datos e información disponible, que cumpla con los requisitos de actualización, seguridad, oportunidad y accesibilidad, para que el docente pueda desarrollar las estrategias adecuadas de aprendizaje (Castelló y Cladellas, 2013). Por su parte, el aprendizaje profesional corresponde al proceso formativo de los docentes y la actualización del conocimiento utilizado en las prácticas docentes que se desarrollan en las aulas de clase. Lo anterior está estrechamente ligado con la personalización. Es decir, el rol del docente y su foco principal de atención, debe estar centrado en mantener actualizada sus prácticas docentes en función de la personalización del aprendizaje y la incorporación de estrategias didácticas orientadas al aprendizaje de cada estudiante (Puentes et al., 2014).

Sin embargo, el docente no es el único actor que debe adaptarse a este cambio de paradigma, la institución de educación superior también forma parte este compromiso, creando las condiciones necesarias para la implementación de las TIC en los procesos formativos, a través de la revisión y ajuste de los currículos para que incorporen las TIC en su desarrollo. Dándole características particulares (personalización), que tienen por propósito comprometer recursos para la expansión y mejora de los activos tecnológicos, estimular el uso de plataformas virtuales y facilitar la interacción entre docentes y estudiantes, con miras a la generación de más eficientes entornos para el aprendizaje (Avello y Duart, 2016).

\section{CONCLUSIONES}

De acuerdo al trabajo presentado y a los resultados obtenidos, se puede afirmar que las TIC han dinamizado de manera notoria los procesos en diversos campos, partiendo por las mejoras insertadas en sistemas de producción, salud y transporte. Al mismo tiempo, aspectos como el educativo han sufrido un impacto relevante, pues con las herramientas derivadas de los procesos tecnológicos y el manejo de la información, se ha logrado promover el nuevo conocimiento lo que a su vez estimula el crecimiento social y económico en la sociedad en general, este último aspecto no solo se observa en el país sino a nivel universal, por lo cual las TIC son consideradas como un componente de gran protagonismo para los aspectos de interés general.

Así las cosas, se puede apreciar a partir de la revisión realizada que la transformación que las TIC han incorporado a los ámbitos educativos ha sido integral, pues no solo ha promovido nuevos espacios o ambientes, sino también que ha posibilitado la generación de nuevo conocimiento y su difusión por mecanismos diversos. Aspectos como los citados se estiman relevantes para el impulso de la economía, pues 
en la medida que en los entornos académicos se alcanzan avances importantes y de representación, los mismos son replicados en los procesos productivos, de servicios y de comercio que impactan de forma directa el desarrollo del país, una vez que los profesionales egresan de las diferentes instituciones y corporaciones y ponen a disposición de la comunidad que integran, todos sus talentos y conocimientos.

\section{REFERENCIAS}

Adell, J., S. Mengual-Andrés y R. Roig-Vila, Presentación del Monográfico, Webquest: 20 años utilizando Internet como recurso para el aula, Edutec. Revista Electrónica de Tecnología Educativa, (52), (2015)

Albion, P.R., J. Tondeur, A. Forkosh-Baruch y J. Peeraer, Teachers' professional development for ICT integration: Towards a reciprocal relationship between research and practice, Education and Information Technologies, 20(4), 655-673 (2015)

Arancibia, M., C.P. Soto y P. Contreras, Concepciones del profesor sobre el uso educativo de las tecnologías de la información y la comunicación (TIC) asociadas a procesos de enseñanza-aprendizaje en el aula escolar, Estudios pedagógicos (Valdivia), 36(1), 23-51 (2010)

Asongu, S.A. y S. Le-Roux, Enhancing ICT for inclusive human development in Sub-Saharan Africa, Technological Forecasting and Social Change, 118, 44-54 (2017)

Ausín, V., V. Abella, V. Delgado y D. Hortigüela, Aprendizaje Basado en Proyectos a través de las TIC: Una Experiencia de Innovación Docente desde las Aulas Universitarias, Formación Universitaria, 9(3), 31-38 (2016)

Avello, R. y J.M. Duart, Nuevas tendencias de aprendizaje colaborativo en e-learning: Claves para su implementación efectiva, Estudios pedagógicos (Valdivia), 42(1), 271-282 (2016)

Avgerou, C., H. Niall y L. Renata, La Rovere, Growth in ICT uptake in developing countries: new users, new uses, New challenges, 329-333 (2016)

Baldassar, L., M. Nedelcu, L. Merla y R. Wilding, ICT-based co-presence in transnational families and communities: challenging the premise of face-to-face proximity in sustaining relationships, Global Networks, 16(2), 133-144 (2016)

Baller, S., S. Dutta y B. Lanvin, Global information technology report 2016, Geneva, Ouranos (2016)

Bejarano, A., J. Angarita y C. Velandia, Implicaciones pedagógicas del uso de las TIC's en la educación superior, Revista de Tecnología, 12(3), 36-56 (2013)

Bello, E.O.G., Digital skills in young people entering the university: realities to innovate in university education, RIDE Revista Iberoamericana para la Investigación y el Desarrollo Educativo, 8(16), 670-687 (2018)

Boyd, D., It's complicate: The social lives of networked teens, New Haven: Yale University Press, ISBN: 978-0-300-199000, p. 281 (2015)

Cabero, J., Los retos de la integración de las TIC's en los procesos educativos, Límites y posibilidades, Perspectiva Educacional, Formación de Profesores, 49 (1), 32-61 (2010)

Castelló, A. y R. Cladellas, La evaluación de la comprensión en el aprendizaje: El empleo de las TIC en el análisis de estructuras de conocimiento, Estudios pedagógicos (Valdivia), 39 (Especial), 41-57 (2013)

De la Hoz-Correa, E., O. Martinez-Palmera y E. De la Hoz-Franco, Innovación desde lo virtual: las TIC y su poder de transformación en las nuevas formas de enseñar y aprender en la Universidad de la Costa, Tercera Conferencia de Directores de Tecnología de Información, TICAL 2013 Gestión de las TIC para la Investigación y la Colaboración, Cartagena de Indias 8 y 9 de Julio de 2013 (2014)

De Witte, K. y N. Rogge, Does ICT matter for effectiveness and efficiency in mathematics education? Computers and Education, 75, 173-184 (2014)

Garcia-Gomez, S., R. Ordonez-Sierra, E. Vinuesa y R. Izquierdo, Vocational education and training students' families' expectations about their future employment, Education Policy Analysis Archives, 24(117), 1-28 (2016)

Hernández, H., D. Martinez y J. Rodríguez, Gestión de la calidad aplicada en el mejoramiento del sector universitario, Revista Espacios, 38(20), (2017)

Herrera, A.M., Una mirada reflexiva sobre las TIC en Educación Superior, Revista electrónica de investigación educativa, 17(1), 1-4 (2015)

Jin, S. y C.M. Cho, Is ICT a new essential for national economic growth in an information society? Government Information Quarterly, 32(3), 253-260 (2015)

Koh, J.H.L., C.S. Chai, W. Benjamin y H.Y. Hong, Technological Pedagogical Content Knowledge (TPACK) and design thinking: A framework to support ICT lesson design for 21st century learning, The Asia-Pacific Education Researcher, 24(3), 535-543 (2015)

Liyanagunawardena, T.R., S. Williams y A.A. Adams, The impact and reach of MOOCs: a developing countries' perspective, eLearning Papers, 38-46 (2014)

López, R.R. y M.W. Hernández, Principios para elaborar un modelo pedagógico universitario basado en las TIC, Estado del arte, EPISTEME - Revista digital de ciencia, Tecnología e Innovación, 3(4), (2016) 
López, S.U., Levels of integration of ICT in the curriculum: a theoretical approach/Dimensions de l'intégration des TIC dans le programme d'études: une approche théorique, Revista Interuniversitaria, 28(1), 209 (2016)

Mac Callum, K. y L. Jeffrey, Comparing the role of ICT literacy and anxiety in the adoption of mobile learning, Computers in Human Behavior, 39, 8-19 (2014)

Marín, F., A. Inciarte, H. Hernández y R. Pitre, Estrategias de las Instituciones de Educación Superior para la Integración de las Tecnología de la Información y la Comunicación y de la Innovación en los Procesos de Enseñanza, Un Estudio en el Distrito de Barranquilla, Colombia, Formación Universitaria, 10(6), (2017)

Molina, A.M., L. Roque y otros cuatro autores, El proceso de comunicación mediado por las tecnologías de la información, Ventajas y desventajas en diferentes esferas de la vida social, MediSur, 13(4), 481-493 (2015)

Pérez-López, R.J., J.E. Olguín-Tiznado y otros tres autores, The Role of Planning and Implementation of ICT in Operational Benefits, Sustainability, 10(7), 2261 (2018)

Pinto, A.C., E. De la Hoz-Franco y D.C. Pinto, Las redes de sensores inalámbricos y el internet de las cosas, Inge CUC, 8(1), 163-172 (2012)

Prensky, M., Digital Natives, Digital Immigrants, On the Horizon - MCB University Press, 9(5), 1-6 (2001)

Puentes, A., R. Roig, S. Sanhueza y M. Friz, Concepciones sobre las Tecnologías de la Información y la Comunicación (TIC) y sus implicaciones educativas: Un estudio exploratorio con profesorado de la provincia de Ñuble, Chile, Revista Iberoamericana de Ciencia, Tecnología y Sociedad - CTI, 8(22), 75-88 (2014)

Recalde-Viana, M., C. Sádaba-Chalezquer y E. Gutiérrez-García, Telecommunications Industry Contributions to Child Online Protection, Comunicar, 23(45), 179-186 (2015)

Roldán, M.A., J. Giraldo y A. Betancur, State of the art and methodological approach to product innovation evaluation in organizations of the telecommunications industry - Estado del arte y aproximación metodológica a la evaluación de la innovación de productos en organizaciones del sector de las telecomunicaciones, Actas de Ingeniería, 2, 210-218 (2016)

Sanz, D.A. y T.J. Crissien-Borrero, Responsabilidad en las instituciones de educación superior, Cultura, Educación y Sociedad, 3(1), 147-156 (2012)

Sarduy, C.J.R.G. y C.P.R.V. Felipe, La universidad en la transformación hacia las redes eléctricas inteligentes en América Latina, Universidad y Sociedad, 6(2) (2014)

Sarnou, H., ICTs Use on Linguistic Change and Identity, Procedia-Social and Behavioral Sciences, 195, 850-855 (2015)

UNESCO, Enfoques estratégicos sobre las TICs en educación en America Latina y el Caribe (2013)

Zempoalteca, B., J.F. Barragán, J. González y T. Guzmán, Formación en TIC y competencia digital en la docencia en instituciones públicas de educación superior, Apertura (Guadalajara, Jal.), 9(1), 80-96 (2017) 